\title{
THE STRUCTURE OF HARMONIC MORPHISMS WITH TOTALLY GEODESIC FIBRES
}

\author{
M. T. MUSTAFA*
}

\begin{abstract}
The structure of local and global harmonic morphisms between Riemannian manifolds, with totally geodesic fibres, is investigated. It is shown that non-positive curvature of the domain obstructs the existence of global harmonic morphisms with totally geodesic fibres and the only such maps from compact Riemannian manifolds of non-positive curvature are, up to a homothety, totally geodesic Riemannian submersions. Similar results are obtained for local harmonic morphisms with totally geodesic fibres from open subsets of non-negatively curved compact and non-compact manifolds. During the course, we prove non-existence of submersive harmonic morphisms with totally geodesic fibres from some important domains, for instance from compact locally symmetric spaces of non-compact type and open subsets of symmetric spaces of compact type.
\end{abstract}

\section{INTRODUCTION}

Harmonic morphisms are maps between Riemannian manifolds which preserve germs of harmonic functions, i.e. these (locally) pull back real-valued harmonic functions to real-valued harmonic functions. These are characterized as a subclass of harmonic maps, precisely, these are harmonic maps which are horizontally (weakly) conformal. What is special about this characterization is that it endows harmonic morphisms with analytic as well as geometric properties. On the other hand, it puts strong restrictions on their existence as solutions of an over-determined system of partial differential equations. The purpose of this article is to study questions related to the existence and structure of harmonic morphisms, with totally geodesic fibres, from compact and non-compact Riemannian manifolds.

The Bochner technique, in its natural setting, is a method to investigate obstructions to the existence of geometric objects on positively curved compact manifolds. The technique mainly relies on the development of a suitable Laplacian identity and its analysis to explore restrictions on the existence of the objects under study. Following the usual Bochner method, the author developed a Bochner technique for harmonic morphisms in [16] and presented restrictions on the existence of harmonic

1991 Mathematics Subject Classification. 58E20.

Key words and phrases. Harmonic morphisms, Bochner technique.

*Regular Associate of The AS-ICTP. 
morphisms from positively curved compact Riemannian manifolds. This was further extended in [17] to include some non-compact domains.

The conventional Bochner technique, though very powerful, is not a handy tool to explore restrictions on the existence of geometric objects on negatively curved compact domains. Until now, the investigation of general restrictions on harmonic morphisms from compact negatively curved manifolds is limited to the following cases.

Case 1: [7] There exist no non-constant Riemannian submersions $\phi: M^{m} \rightarrow N^{n}$ $(m>n)$ with totally geodesic fibres if $M$ has negative sectional curvature.

Case 2: [2] Every harmonic morphism from the compact quotients of the hyperbolic space $\mathbb{H}^{3}$ to a Riemann surface is constant.

Case 3: [18] Any non-constant submersive harmonic morphism from a compact Riemannian manifold $M^{n+1}$ to $N^{n}$, such that $\operatorname{Ric}^{M}(U, U) \leq 0$ for $U$ vertical, is totally geodesic.

Realizing that all the maps considered above are particular cases of submersive harmonic morphisms with totally geodesic fibres, we take a unified approach and study the restrictions on the existence of harmonic morphisms with totally geodesic fibres and their structure, in case these exist. In order to do so, we develop a variant of the usual Bochner technique by developing a generalized Bochner type formula which leads to obtaining restrictions on the existence of harmonic morphisms, with totally geodesic fibres, from negatively curved compact Riemannian manifolds. These restrictions contain the above results as particular cases. A comparitive study of this generalized Bochner type formula with the usual one [16, Proposition 2.1] provides (local) non-existence results for harmonic morphisms, with totally geodesic fibres, from non-negatively curved Riemannian manifolds. As a final consequence, we obtain a classification of submersive harmonic morphisms, having totally geodesic fibres, from open subsets of $\mathbb{R}^{m}$ to complete manifolds of non-positive scalar curvature.

A conventional remark: The sign convention adopted for the curvature is the one that coincides with the classical curvature tensor i.e. for vector fields $X, Y$, the curvature $\mathbf{R}$ of a connection $\nabla$ is

$$
\mathbf{R}(X, Y)=-\nabla_{X} \nabla_{Y}+\nabla_{Y} \nabla_{X}+\nabla_{[X, Y]}
$$

\section{HARMONIC MORPHISMS}

The formal theory of harmonic morphisms between Riemannian manifolds began with the work of Fuglede [8] and Ishihara [13].

Definition 2.1. A smooth map $\phi: M^{m} \rightarrow N^{n}$ between Riemannian manifolds is called a harmonic morphism if, for every real-valued function $f$ which is harmonic 
on an open subset $U$ of $N$ with $\phi^{-1}(U)$ non-empty, $f \circ \phi$ is a harmonic function on $\phi^{-1}(U)$.

Harmonic morphisms are related to horizontally (weakly) conformal maps which can be defined in the following manner.

For a smooth map $\phi: M^{m} \rightarrow N^{n}$, let $C_{\phi}=\left\{x \in M \mid \operatorname{rank} d \phi_{x}<n\right\}$ be its critical set. The points of the set $M \backslash C_{\phi}$ are called regular points. For each $x \in M \backslash C_{\phi}$, the vertical space at $x$ is defined by $\mathcal{V}_{x}=\operatorname{Ker} d \phi_{x}$. The horizontal space $\mathcal{H}_{x}$ at $x$ is given by the orthogonal complement of $\mathcal{V}_{x}$ in $T_{x} M$.

Definition 2.2. A smooth map $\phi:\left(M^{m}, \mathbf{g}\right) \rightarrow\left(N^{n}, \mathbf{h}\right)$ is called horizontally (weakly) conformal if $d \phi=0$ on $C_{\phi}$ and the restriction of $\phi$ to $M \backslash C_{\phi}$ is a conformal submersion, that is, for each $x \in M \backslash C_{\phi}$, the differential $d \phi_{x}: T_{x}^{H} M \rightarrow T_{\phi(x)} N$ is conformal and surjective. This means that there exists a function $\lambda: M \backslash C_{\phi} \rightarrow \mathbb{R}^{+}$such that

$$
\mathbf{h}(d \phi(X), d \phi(Y))=\lambda^{2} \mathbf{g}(X, Y) \quad \forall X, Y \in T_{x}^{H} M .
$$

By setting $\lambda=0$ on $C_{\phi}$, we can extend $\lambda: M \rightarrow \mathbb{R}_{0}^{+}$to a continuous function on $M$ such that $\lambda^{2}$ is smooth. The extended function $\lambda: M \rightarrow \mathbb{R}_{0}^{+}$is called the dilation of the map.

Let $\operatorname{grad}_{\mathcal{H}} \lambda^{2}$ and $\operatorname{grad}_{\mathcal{V}} \lambda^{2}$ denote the horizontal and vertical projections of $\operatorname{grad} \lambda^{2}$.

Definition 2.3. A smooth map $\phi: M^{m} \rightarrow N^{n}$ is called horizontally homothetic if it is a horizontally conformal submersion whose dilation is constant along the horizontal curves i.e. $\operatorname{grad}_{\mathcal{H}} \lambda^{2}=0$.

Recall that a map $\phi: M^{m} \rightarrow N^{n}$ is said to be harmonic if it extremizes the associated energy integral $E(\phi)=\frac{1}{2} \int_{\Omega}\left\|\phi_{*}\right\|^{2} d v^{M}$ for every compact domain $\Omega \subset M$. It is well-known that a map $\phi$ is harmonic if and only if its tension field vanishes.

Harmonic morphisms can be viewed as a subclass of harmonic maps in the light of the following characterization, obtained in $[8,13]$.

A smooth map is a harmonic morphism if and only if it is harmonic and horizontally (weakly) conformal.

The following result of Baird-Eells [3, Riemannian case] and Gudmundsson [11, semiRiemannian case] reflects a significant geometric feature of harmonic morphisms.

Theorem 2.4. Let $\phi: M^{m} \rightarrow N^{n}$ be a horizontally conformal submersion with dilation $\lambda$. If

(1) $n=2$, then $\phi$ is a harmonic map if and only if it has minimal fibres.

(2) $n \geq 3$, then two of the following imply the other,

(a) $\phi$ is a harmonic map 
(b) $\phi$ has minimal fibres

(c) $\phi$ is horizontally homothetic.

The notion of horizontally conformal maps is a natural generalization of Riemannian submersions. The fundamental equations of Riemannian submersions were generalized for horizontally conformal submersions in [10]. We state those results which will be needed in the proof of Bochner formula and refer to [10] for complete details.

If $T$ and $A$ denote the standard fundamental tensors of a horizontally conformal submersion $\phi$ then the relation of the integrability of horizontal distribution with the tensor $A$ is given by the following expression.

$$
A_{X} Y=\frac{1}{2}\left\{\mathcal{V}[X, Y]-\lambda^{2} \mathbf{g}(X, Y) \operatorname{grad}_{\mathcal{V}} \frac{1}{\lambda^{2}}\right\} \quad(X, Y \text { horizontal }) .
$$

Moreover, the mixed sectional curvatures of the domain satisfy the following relation.

Proposition 2.5. [10] Let $\phi:\left(M^{m}, \mathbf{g}\right) \rightarrow\left(N^{n}, \mathbf{h}\right)$ be a horizontally conformal submersion with totally geodesic fibres. If $X, Y$ are horizontal vectors and $U, V$ are vertical vectors then

$$
\begin{aligned}
\mathbf{g}\left(\mathbf{R}^{\mathbf{M}}(X, U) Y, V\right) & =\mathbf{g}\left(\left(\nabla_{U} A\right)_{X} Y, V\right)+\mathbf{g}\left(A_{X} U, A_{Y} V\right) \\
& +\lambda^{2} \mathbf{g}\left(A_{X} Y, U\right) \mathbf{g}\left(\operatorname{grad}_{\mathcal{V}} \frac{1}{\lambda^{2}}, V\right)
\end{aligned}
$$

where $\lambda$ is the dilation.

For the fundamental results and properties of harmonic morphisms, the reader is referred to $[1,6,8,19]$ and for an updated online bibliography to [12].

\section{The Bochner type Formula}

In this section we develop the generalized Bochner type formula, which will be the main tool in the next section. To establish the formula, we consider a horizontally homothetic map with totally geodesic fibres (equivalently a submersive harmonic morphism with totally geodesic fibres) and compute the Laplacian of the dilation.

Proposition 3.1. Let $\phi:\left(M^{m}, \mathbf{g}\right) \rightarrow\left(N^{n}, \mathbf{h}\right)$ be a non-constant horizontally homothetic map with totally geodesic fibres. If $\lambda$ denotes the dilation of $\phi$ then

$$
\begin{aligned}
\frac{n}{2} \Delta \lambda^{2} & =\lambda^{2} \sum_{\alpha=1}^{n} \sum_{i=n+1}^{m}\left\{\mathbf{g}\left(A_{e_{\alpha}} e_{i}, A_{e_{\alpha}} e_{i}\right)-\mathbf{g}\left(\mathbf{R}^{\mathbf{M}}\left(e_{\alpha}, e_{i}\right) e_{\alpha}, e_{i}\right)\right\} \\
& +\frac{n(n-4)}{4} \lambda^{6} \mathbf{g}\left(\operatorname{grad}_{\mathcal{V}} \frac{1}{\lambda^{2}}, \operatorname{grad}_{\mathcal{V}} \frac{1}{\lambda^{2}}\right)
\end{aligned}
$$

where $\left(e_{\alpha}\right)_{\alpha=1}^{n}$ and $\left(e_{i}\right)_{i=n+1}^{m}$ are local orthonormal frames for the horizontal and vertical distributions respectively. 
Proof. Taking the Laplacian on functions as

$$
\Delta f=-\operatorname{div}(\operatorname{grad} f)
$$

we have, for the dilation $\lambda$ of $\phi$

$$
\begin{aligned}
\frac{1}{\lambda^{2}} \Delta \lambda^{2}= & -\lambda^{2} \Delta \frac{1}{\lambda^{2}}+2 \mathbf{g}\left(\operatorname{grad}_{\mathcal{V}} \lambda^{2}, \operatorname{grad}_{\mathcal{V}} \frac{1}{\lambda^{2}}\right) \\
= & -\lambda^{2} \Delta \frac{1}{\lambda^{2}}-2 \lambda^{4} \mathbf{g}\left(\operatorname{grad}_{\mathcal{V}} \frac{1}{\lambda^{2}}, \operatorname{grad}_{\mathcal{V}} \frac{1}{\lambda^{2}}\right) \\
= & \lambda^{2} \sum_{\alpha=1}^{n} \mathbf{g}\left(\nabla_{e_{\alpha}} \operatorname{grad}_{\mathcal{V}} \frac{1}{\lambda^{2}}, e_{\alpha}\right)+\lambda^{2} \sum_{i=n+1}^{m} \mathbf{g}\left(\nabla_{e_{i}} \operatorname{grad}_{\mathcal{V}} \frac{1}{\lambda^{2}}, e_{i}\right) \\
& -2 \lambda^{4} \mathbf{g}\left(\operatorname{grad}_{\mathcal{V}} \frac{1}{\lambda^{2}}, \operatorname{grad}_{\mathcal{V}} \frac{1}{\lambda^{2}}\right) .
\end{aligned}
$$

Since

we have

$$
\mathbf{g}\left(\nabla_{e_{\alpha}} \operatorname{grad}_{\mathcal{V}} \frac{1}{\lambda^{2}}, e_{\alpha}\right)=-\mathbf{g}\left(\operatorname{grad}_{\mathcal{V}} \frac{1}{\lambda^{2}}, A_{e_{\alpha}} e_{\alpha}\right)
$$

$$
\frac{1}{\lambda^{2}} \Delta \lambda^{2}=\frac{(n-4)}{2} \lambda^{4} \mathbf{g}\left(\operatorname{grad}_{\mathcal{V}} \frac{1}{\lambda^{2}}, \operatorname{grad}_{\mathcal{V}} \frac{1}{\lambda^{2}}\right)+\lambda^{2} \sum_{i=n+1}^{m} \mathbf{g}\left(\nabla_{e_{i}} \operatorname{grad}_{\mathcal{V}} \frac{1}{\lambda^{2}}, e_{i}\right)
$$

Now a straight forward computation using Equation 2.1 and the easily seen relation $\mathbf{g}\left(\left(\nabla_{e_{i}} A\right)_{e_{\alpha}} e_{\alpha}, e_{i}\right)=\mathbf{g}\left(\nabla_{e_{i}}\left(A_{e_{\alpha}} e_{\alpha}\right), e_{i}\right)$ implies that

$\lambda^{2} \sum_{i=n+1}^{m} \mathbf{g}\left(\nabla_{e_{i}} \operatorname{grad}_{\mathcal{V}} \frac{1}{\lambda^{2}}, e_{i}\right)=-2 \sum_{i=n+1}^{m} \mathbf{g}\left(\left(\nabla_{e_{i}} A\right)_{e_{\alpha}} e_{\alpha}, e_{i}\right)-2 \lambda^{2} \sum_{i=n+1}^{m} \mathbf{g}\left(A_{e_{\alpha}} e_{\alpha}, e_{i}\right) \mathbf{g}\left(\operatorname{grad}_{\mathcal{V}} \frac{1}{\lambda^{2}}, e_{i}\right)$.

Using Equation 3.3 and Equation 2.2 we can write Equation 3.2 as

$$
\begin{aligned}
\frac{1}{2} \Delta \lambda^{2}= & \frac{(n-4)}{2} \lambda^{6} \mathbf{g}\left(\operatorname{grad}_{\mathcal{V}} \frac{1}{\lambda^{2}}, \operatorname{grad}_{\mathcal{V}} \frac{1}{\lambda^{2}}\right)+\lambda^{2} \sum_{i=n+1}^{m} \mathbf{g}\left(A_{e_{\alpha}} e_{i}, A_{e_{\alpha}} e_{i}\right) \\
& -\lambda^{2} \sum_{i=n+1}^{m} \mathbf{g}\left(\mathbf{R}^{\mathbf{M}}\left(e_{\alpha}, e_{i}\right) e_{\alpha}, e_{i}\right)
\end{aligned}
$$

Since the above identity holds for each $e_{\alpha}$ for $\alpha=1, \ldots, n$, therefore, summing over $\alpha$ completes the proof.

The above formula will naturally be useful to obtain consequences for maps from compact Riemannian manifolds. For the general domains, a comparison of this Bochner type formula with the Weitzenböck formula of [16, Proposition 2.1] yields the following identity.

Proposition 3.2. If the Riemannian manifolds $M^{m}, N^{n}$ admit a submersive harmonic morphism $\phi: M^{m} \rightarrow N^{n}$ having totally geodesic fibres then the following 
identity is satisfied.

$$
\begin{gathered}
-\|\nabla d \phi\|^{2}+\lambda^{4} \mathbf{S c a l}^{\mathbf{N}}-\lambda^{2} \sum_{\alpha=1}^{n} \sum_{\beta=1}^{n} \mathbf{g}\left(\mathbf{R}^{\mathbf{M}}\left(e_{\alpha}, e_{\beta}\right) e_{\alpha}, e_{\beta}\right) \\
=\lambda^{2} \sum_{\alpha=1}^{n} \sum_{i=n+1}^{m} \mathbf{g}\left(A_{e_{\alpha}} e_{i}, A_{e_{\alpha}} e_{i}\right)+\frac{n(n-4)}{2} \lambda^{6} \mathbf{g}\left(\operatorname{grad}_{\mathcal{V}} \frac{1}{\lambda^{2}}, \operatorname{grad}_{\mathcal{V}} \frac{1}{\lambda^{2}}\right)
\end{gathered}
$$

where $\lambda$ is the dilation of $\phi$ and $\left(e_{\alpha}\right)_{\alpha=1}^{n},\left(e_{i}\right)_{i=n+1}^{m}$ are local orthonormal frames for the horizontal, vertical distributions respectively.

Proof. The Weitzenböck formula of [16, Proposition 2.1] says that the dilation $\lambda$ of a harmonic morphism satisfies

$$
\frac{n}{2} \Delta \lambda^{2}=-\|\nabla d \phi\|^{2}+\lambda^{4} \mathbf{S c a l}^{\mathbf{N}}-\lambda^{2} \sum_{\alpha=1}^{n} \mathbf{R i c}^{M}\left(e_{\alpha}, e_{\alpha}\right)
$$

Writing

$$
\sum_{\alpha=1}^{n} \mathbf{R i c}^{M}\left(e_{\alpha}, e_{\alpha}\right)=\sum_{\alpha=1}^{n} \sum_{\beta=1}^{n} \mathbf{g}\left(\mathbf{R}^{\mathbf{M}}\left(e_{\alpha}, e_{\beta}\right) e_{\alpha}, e_{\beta}\right)+\sum_{\alpha=1}^{n} \sum_{i=n+1}^{m} \mathbf{g}\left(\mathbf{R}^{\mathbf{M}}\left(e_{\alpha}, e_{i}\right) e_{\alpha}, e_{i}\right)
$$

and comparing above Weitzenböck formula with Equation 3.1 gives the required identity.

\section{Applications to HARMONiC MORPhisms With TOTALly GEODESiC FiBRES}

This section is devoted to analysis of the structure of globally as well as locally defined harmonic morphisms with totally geodesic fibres. Throughout the section we assume the following range of indices:

$$
1 \leq \alpha, \beta \leq n ; \quad n+1 \leq i, j \leq m
$$

Moreover, $\left(e_{\alpha}\right)_{\alpha=1}^{n},\left(e_{i}\right)_{i=n+1}^{m}$ will denote local orthonormal frames for the horizontal, vertical distributions, respectively, induced by a horizontally conformal map $\phi: M^{m} \rightarrow N^{n}$.

4.1. Global harmonic morphisms with totally geodesic fibres. The main result, obtained by applying Proposition 3.1, is that the negative curvature of compact domains obstructs the existence of harmonic morphisms with totally geodesic fibres, thus making the class of such harmonic morphisms very restricted on domains of nonpositive curvature. Combining the results of this section with Theorem 2.5 of [16] gives a much clearer picture of the structure of harmonic morphisms from compact manifolds, having totally geodesic fibres (cf. Corollary 4.5).

Firstly we consider harmonic morphisms of co-dimension $>1$. 
Theorem 4.1. Let $\phi: M^{m} \rightarrow N^{n}(n \geq 4, m \geq n+2)$ be a non-constant submersive harmonic morphism from a compact Riemannian manifold such that the sectional curvature $\mathbf{K}^{\mathbf{M}}\left(e_{\alpha} \wedge e_{i}\right) \leq 0 \forall \alpha, i$.

(1) If $\phi$ has totally geodesic fibres then it has constant dilation and integrable horizontal distribution i.e. up to a homothety it is a totally geodesic Riemannian submersion.

(2) If $\mathbf{K}^{\mathbf{M}}\left(e_{\alpha} \wedge e_{i}\right)<0$ at some point for at least one pair of $\alpha, i$ then there are no non-constant submersive harmonic morphisms with totally geodesic fibres from $M$ to $N$.

Proof. ¿From Stokes' theorem and Proposition 3.1

$$
\begin{aligned}
0 & =\int_{M}\left[\lambda^{2} \sum_{\alpha=1}^{n} \sum_{i=n+1}^{m}\left\{\mathbf{g}\left(A_{e_{\alpha}} e_{i}, A_{e_{\alpha}} e_{i}\right)-\mathbf{g}\left(\mathbf{R}^{\mathbf{M}}\left(e_{\alpha}, e_{i}\right) e_{\alpha}, e_{i}\right)\right\}\right] v^{M} \\
& +\frac{n(n-4)}{4} \int_{M} \lambda^{6} \mathbf{g}\left(\operatorname{grad}_{\mathcal{V}} \frac{1}{\lambda^{2}}, \operatorname{grad}_{\mathcal{V}} \frac{1}{\lambda^{2}}\right) v^{M} .
\end{aligned}
$$

Now the hypothesis and a standard Bochner type argument forces each term on the right hand side of Equation 3.1 to vanish, which completes the proof.

For harmonic morphisms with one dimensional fibres, the condition on the sectional curvature can be replaced by Ricci curvature to obtain better consequences.

Corollary 4.2. Let $\phi: M^{n+1} \rightarrow N^{n}(n \geq 4)$ be a non-constant harmonic morphism between compact Riemannian manifolds. Let $\left.\mathbf{R i c}{ }^{\mathbf{M}}\right|_{\mathcal{V}}$ denote the Ricci curvature of $M$ restricted to the fibres. i.e.

$$
\left.\operatorname{Ric}^{\mathbf{M}}\right|_{\mathcal{V}}=\operatorname{Ric}^{\mathbf{M}}\left(e_{n+1}, e_{n+1}\right)
$$

for a vertical unit vector $e_{n+1}$.

(1) If $\left.\mathbf{R i c}^{\mathbf{M}}\right|_{\mathcal{V}} \leq 0$ then, up to a homothety, $\phi$ is a totally geodesic Riemannian submersion.

(2) If $\left.\mathbf{R i c}^{\mathbf{M}}\right|_{\mathcal{V}}<0$ at some point then $\phi$ does not exist.

(3) In particular, if $M$ is Ricci-flat then, up to a homothety, $\phi$ is a totally geodesic Riemannian submersion, and the fibres, the horizontal submanifolds and $N$ are all Ricci-flat.

Proof. First notice that $\phi: M^{n+1} \rightarrow N^{n}(n \geq 4)$ is submersive, as shown in [2]. Moreover, the fibres are automatically totally geodesic. Now Parts 1,2 are similar to the proof of Theorem 4.1, since

$$
\mathbf{R i c}^{\mathbf{M}}\left(e_{n+1}, e_{n+1}\right)=\sum_{\alpha=1}^{n} \mathbf{g}\left(\mathbf{R}^{\mathbf{M}}\left(e_{\alpha}, e_{n+1}\right) e_{\alpha}, e_{n+1}\right) .
$$


For Part 3 we see that $\phi$, being totally geodesic Riemannian submersion, makes the horizontal and vertical foliations Riemannian with totally geodesic leaves. Hence, the Ricci curvatures of the fibres, the horizontal submanifolds and the target manifold vanish because of Ricci-flatness of $M$.

The reader is referred to [18, Corollary 3.6] where Part 1 of the above result is proved by a different approach.

Next, we discuss examples satisfying the hypothesis of Theorem 4.1.

Corollary 4.3. Let $M^{n+1}$ be a compact Riemannian manifold. Then there exists a metric $\mathrm{g}$ on $M$ such that there are no non-constant harmonic morphisms $\phi$ : $\left(M^{n+1}, \mathbf{g}\right) \rightarrow N^{n}$ for $(n \geq 4)$.

Proof. Due to Lohkamp [15, Corollary 5.2], any manifold of dimension $\geq 3$ carries a Riemannian metric of negative Ricci curvature and hence, from above we have the non-existence result.

By considering compact locally symmetric spaces of non-compact type, we get a number of examples where Theorem 4.1 can be applied to obtain restrictions or nonexistence results for harmonic morphisms with totally geodesic fibres.

\section{Corollary 4.4.}

(1) There are no non-constant submersive harmonic morphisms with totally geodesic fibres from compact locally symmetric spaces $M^{m}(m>n)$ of noncompact type and rank 1 to any Riemannian manifold $N^{n}(n \geq 4)$.

(2) Every non-constant submersive harmonic morphism with totally geodesic fibres, from a compact irreducible locally symmetric space $M^{m}(m>n)$ of non-compact type and rank $\geq 2$ to any Riemannian manifold $N^{n}(n \geq 4)$ is, up to a homothety, a totally geodesic Riemannian submersion.

Proof.

(1) Follows from the fact that all compact locally symmetric spaces $M^{m} m \geq 3$ of non-compact type and rank 1 have negative sectional curvature.

(2) Since every compact irreducible locally symmetric space $M^{m}$ of non-compact type and rank $\geq 2$ has the sectional curvature $\mathbf{K} \leq 0$ (but $\mathbf{K} \nless 0$ ), the proof follows from above.

We refer the reader to [11] where, in contrast to above result, Gudmundsson has given an affirmative answer to the global existence question for harmonic morphisms, 
from irreducible symmetric spaces of non-compact type and rank 1, with values in complex plane.

If we focus only on manifolds whose sectional curvatures do not change sign i.e either $\mathbf{K} \leq 0$ everywhere or $\mathbf{K} \geq 0$ everywhere. Then combining Theorem 4.1 with [16, Theorem 2.5] we have the following picture of the structure of submersive harmonic morphisms $\phi$ with totally geodesic fibres from compact Riemannian manifolds $M^{m}$ to compact Riemannian manifolds $N^{n}$ such that $n \geq 4$ and $m>n$ and the sectional curvatures of $M, N$ do no change sign.

Corollary 4.5. Let $M^{m}, N^{n}$ be compact Riemannian manifolds with $n \geq 4$ and $m>n$.

(1) If $\mathbf{K}^{\mathbf{M}} \leq 0$ and $\mathbf{K}^{\mathbf{M}}<0$ at some point then there are no non-constant submersive harmonic morphisms with totally geodesic fibres.

(2) If $\mathbf{K}^{\mathbf{M}} \equiv 0$ then every submersive harmonic morphism with totally geodesic fibres is, up to a homothety, a totally geodesic Riemannian submersion, and the fibres, the horizontal submanifolds and the target manifold $N$ are all flat.

(3) If $\mathbf{K}^{\mathbf{M}} \geq 0$ and $\mathbf{K}^{\mathbf{M}} \not \equiv 0$ then only possible non-constant submersive harmonic morphisms $\phi$ with totally geodesic fibres are when $\mathbf{S c a l}^{\mathbf{N}} \geq 0$ and $\mathbf{S c a l}^{\mathbf{N}} \not \equiv$ 0. In which case, either the horizontal distribution can be integrable or the dilation can be constant but both cannot occur.

4.2. Local harmonic morphisms with totally geodesic fibres. The obstruction theory for local harmonic morphisms with totally geodesic fibres has an interesting contrast to the global case in the sense that instead of negative curvature it is the positive curvature which imposes restrictions on the existence of local harmonic morphisms with totally geodesic fibres.

For a horizontally conformal submersion $\phi:\left(M^{m}, \mathbf{g}\right) \rightarrow N^{n}$, let $\mathbf{S c a l}^{\mathbf{M}_{\mathbf{H}}}$ be the curvature given by

$$
\mathbf{S c a l}^{\mathbf{M}_{\mathbf{H}}}=\sum_{\alpha, \beta=1}^{n} \mathbf{g}\left(\mathbf{R}^{\mathbf{M}}\left(e_{\alpha}, e_{\beta}\right) e_{\alpha}, e_{\beta}\right)
$$

for a local orthonormal frame $\left(e_{\alpha}\right)_{\alpha=1}^{n}$ of the horizontal distribution. When the horizontal distribution is integrable and totally geodesic $\mathbf{S c a l}^{\mathbf{M}_{\mathbf{H}}}$ coincides with the scalar curvature $\mathbf{S c a l}^{\mathcal{H}}$ of the horizontal submanifolds.

Theorem 4.6. Let $U$ be a connected open subset of a Riemannian manifold $M, N^{n}$ $(n \geq 4)$ be a Riemannian manifold with $\mathbf{S c a l}^{\mathbf{N}} \leq 0$ and $\phi: U \rightarrow N^{n}$ be a nonconstant submersive harmonic morphism with totally geodesic fibres. If $\mathbf{S c a l}^{\mathbf{M}_{\mathbf{H}}} \geq 0$ then, up to a homothety, $\phi$ is a Riemannian submersion with totally geodesic fibres and integrable horizontal distribution. Furthermore, 
(1) The scalar curvature of the horizontal submanifolds is zero.

(2) If $\mathbf{S c a l}^{\mathbf{N}}<0$ at some point, then such $\phi$ cannot exist.

Proof. The hypothesis combined with Proposition 3.2 makes each term in Equation 3.5 vanish, which makes the dilation constant and the tensor $A \equiv 0$. The rest is immediate, since the horizontal foliation becomes Riemannian with totally geodesic leaves.

Theorem 4.6 enables us to find local analogue of the results of [16] where restrictions on the existence of global harmonic morphisms from Riemannian symmetric spaces of compact type were obtained.

Corollary 4.7. Let $M^{m}$ be a irreducible Riemannian symmetric space of compact type, $U$ a connected open subset of $M$ and $N^{n}(n \geq 4)$ any Riemannian manifold.

(1) If $\mathbf{S c a l}^{\mathbf{N}} \leq 0$ but $\mathbf{S c a l}^{\mathbf{N}} \not \equiv 0$ then there exists no non-constant submersive harmonic morphism $\phi: U \rightarrow N^{n}$ with totally geodesic fibres.

(2) In particular, there exists a metric $\mathbf{h}$ on $N^{n}$ such that there are no nonconstant submersive harmonic morphisms $\phi: U \rightarrow\left(N^{n}, \mathbf{h}\right)$ having totally geodesic fibres.

(3) If $\mathbf{S c a l}^{\mathbf{N}} \equiv 0$ then every non-constant submersive harmonic morphism $\phi$ : $U \rightarrow N^{n}$ with totally geodesic fibres, is up to a homothety, a totally geodesic Riemannian submersion.

Proof.

(1) The proof follows from the fact that irreducible Riemannian symmetric space of compact type have non-negative sectional curvature.

(2) As $n>3$, there exists a Riemannian metric of negative Ricci curvature on $N^{n}$ by $[15]$.

(3) Immediate from above.

A classification of local and global harmonic morphisms from 3-dimensional simplyconnected space-forms was found in $[4,5]$ which was generalized to higher dimensions in [14] for global harmonic morphisms from $\mathbb{R}^{m}$ having totally geodesic fibres. For local case, a classification of harmonic morphisms, with totally geodesic fibres and integrable horizontal distribution, between higher-dimensional simply-connected spaceforms was obtained in [9]. Here, we extend the classification of [14] to local harmonic morphisms with totally geodesic fibres. 
Corollary 4.8. Let $U$ be a connected open subset of $\mathbb{R}^{m}$ and $\phi: U \rightarrow N^{n}(n \geq 4)$ be a non-constant submersive harmonic morphism with totally geodesic fibres.

(1) If $N$ is complete, simply-connected with $\mathbf{S c a l}^{\mathbf{N}} \leq 0$ then $\phi$ is an orthogonal projection followed by a homothety.

(2) If $\mathbf{S c a l}^{\mathbf{N}}>0$ then $\phi$ has a non-constant dilation.

Proof.

(1) From Theorem 4.6, up to a homothety, $\phi$ is a Riemannian submersion with totally geodesic fibres and integrable horizontal distribution. Therefore, the horizontal submanifolds are totally geodesic and flat, hence $\mathbf{K}^{\mathbf{N}} \equiv 0$, which completes the proof.

(2) If $\phi$ has constant dilation then the horizontal distribution is integrable from Equation 3.1. This makes $\phi$ a totally geodesic map and then Equation 3.5 implies that $\mathbf{S c a l}^{\mathbf{N}} \equiv 0$; a contradiction.

Acknowledgements. This work was carried out during a research visit (under The Associate Scheme) to The Abdus Salam International Centre for Theoretical Physics, Trieste. The author is thankful to The Director of The AS-ICTP for this support. The author would also like to acknowledge the support of KFUPM, Dhahran.

\section{REFERENCES}

[1] P. Baird, Harmonic maps with symmetry, harmonic morphisms, and deformation of metrics, Pitman Research Notes in Mathematics Series 87, Pitman, Boston, London, Melbourne, 1983.

[2] P. Baird, Harmonic morphisms and circle actions on 3- and 4-manifolds, Ann. Inst. Fourier (Grenoble) 40 (1990) 177-212.

[3] P. Baird and J. Eells, A conservation law for harmonic maps, Lecture Notes in Mathematics 894 (1981) 1-25.

[4] P. Baird and J. C. Wood, Bernstein theorems for harmonic morphisms from $\mathbb{R}^{3}$ and $\mathbb{S}^{3}$, Math. Ann. 280 (1988) 579-603.

[5] P. Baird and J. C. Wood, Harmonic morphisms and conformal foliation by geodesics of threedimensional space forms, J. Australian Math. Soc. A51 (1991) 118-153.

[6] P. Baird and J. C. Wood, Harmonic morphisms between Riemannian manifolds, London Math. Soc. Monogr. (N.S.), Oxford University Press No. 29, Oxford Univ. Press (2003).

[7] R. H. Escobales, Riemannian submersions with totally geodesic fibres, J. Diff. Geom. 10 (1975) 253-276.

[8] B. Fuglede, Harmonic morphisms between Riemannian manifolds, Ann. Inst. Fourier (Grenoble) 28 (1978) 107-144.

[9] S. Gudmundsson, Harmonic morphisms between spaces of constant curvature, Proc. Edinburgh Math. Soc. 36 (1992) 133-143.

[10] S. Gudmundsson, The geometry of harmonic morphisms, Ph.D. thesis, University of Leeds (1992).

[11] S. Gudmundsson, On the existence of harmonic morphisms from symmetric spaces of rank one, Manuscripta Math. 93 (1997) 421-433. 
[12] S. Gudmundsson, The Bibliography of Harmonic Morphisms, http://www.maths.lth.se/ matematiklu/personal/sigma/harmonic/bibliography.html

[13] T. Ishihara, A mapping of Riemannian manifolds which preserves harmonic functions, J. Math. Kyoto Univ. 19 (1979) 215-229.

[14] A. Kasue and T. Washio, Growth of equivariant harmonic maps and harmonic morphisms, Osaka J. Math. 27 (1990) 899-928.

[15] J. Lohkamp, Metrics of negative Ricci curvature, Ann. of Math. 140 (1994) 655-683.

[16] M. T. Mustafa, A Bochner technique for harmonic morphisms, J. London Math. Soc. (2) 57 (1998) 746-756.

[17] M. T. Mustafa, Restrictions on harmonic morphisms, Conformal Geometry and Dynamics 3 (1999) 102-115.

[18] R. Pantilie, Harmonic morphisms with one-dimensional fibres, Internat. J. Math. 10 (1999) $457-501$.

[19] J. C. Wood, Harmonic morphisms, foliations and Gauss maps, Complex differential geometry and nonlinear partial differential equations (Providence, R.I.) (Y.T. Siu, ed.), Contemp. Math. 49, Amer. Math. Soc., Providence, R.I., 1986, 145-184.

Department of Mathematical Sciences, King Fahd University of Petroleum \& Minerals, Dhahran 31261, Saudi Arabia

E-mail address: tmustafa@kfupm.edu.sa

E-mail address: mtmustafa@yahoo.com 